\title{
A giant spinal aneurysm with cord and root compression
}

\author{
MOKHTAR A EL MAHDI, MOHAMED A RUDWAN,* SALAH M KHAFFAJI,* \\ FAWZI A JADALLAH
}

From the Department of Neurosurgery and Radiology*, Ibn Sina Hospital, Kuwait

SUMMARY A patient suffering from a giant spinal aneurysm is reported. The diagnosis was made by angiography and dynamic computed tomography. The patient was treated successfully by clipping of the feeding artery.

\begin{abstract}
Very few cases of true spinal aneurysms have been documented, ${ }^{1-8}$ even fewer have been demonstrated by angiography. ${ }^{13-6} \mathrm{We}$ report a case of unruptured giant spinal aneurysm presenting with cord and root compression. An aneurysm of this size and the clinicoradiological presentation have not, to our knowledge, been reported before.
\end{abstract}

\section{Case report}

A 17 year old girl presented with a 4 year history of mild low back pain and occasional right sciatica. Her parents stated that she had only stopped wetting her bed at the age of 15 years, but was still having urgency of micturition. She had a mild scoliotic deformity at the dorsolumbar region, relative weakness of the plantar flexors of the right ankle and absent right ankle jerk. Straight leg raising on the right side was painful and limited to $50^{\circ}$, the right plantar reflex was equivocal and the lower abdominal reflex was absent on the right side. No cafe-au-lait spots were seen and the pulses in both upper and lower limbs were normal.

The plain dorsolumbar radiographs showed scoliosis and a widened canal with scalloping of the posterior surface of D12 vertebra. Metrizamide lumbar myelography showed a complete block with a classic "cap" defect at the upper border of lst lumbar vertebra. Most of the nerve roots of the cauda equina appeared to descend from the left side, suggesting displacement of the conus in this direction by an extramedullary intradural lesion (fig 1). Postmyelography CT showed the upper limit of the lesion at the upper border of D12. It measured $3 \times 3 \times 2 \mathrm{~cm}$, and was filling and expanding the spinal canal at D12 (fig 2).

Address for reprint requests: Dr Mohamed A. Rudwan, Radiology Department, Ibn Sina Hospital, P.O. Box 25427, Safat 13115, Kuwait. Received 13 May 1988 and in revised form 6 October 1988. Accepted 12 November 1988

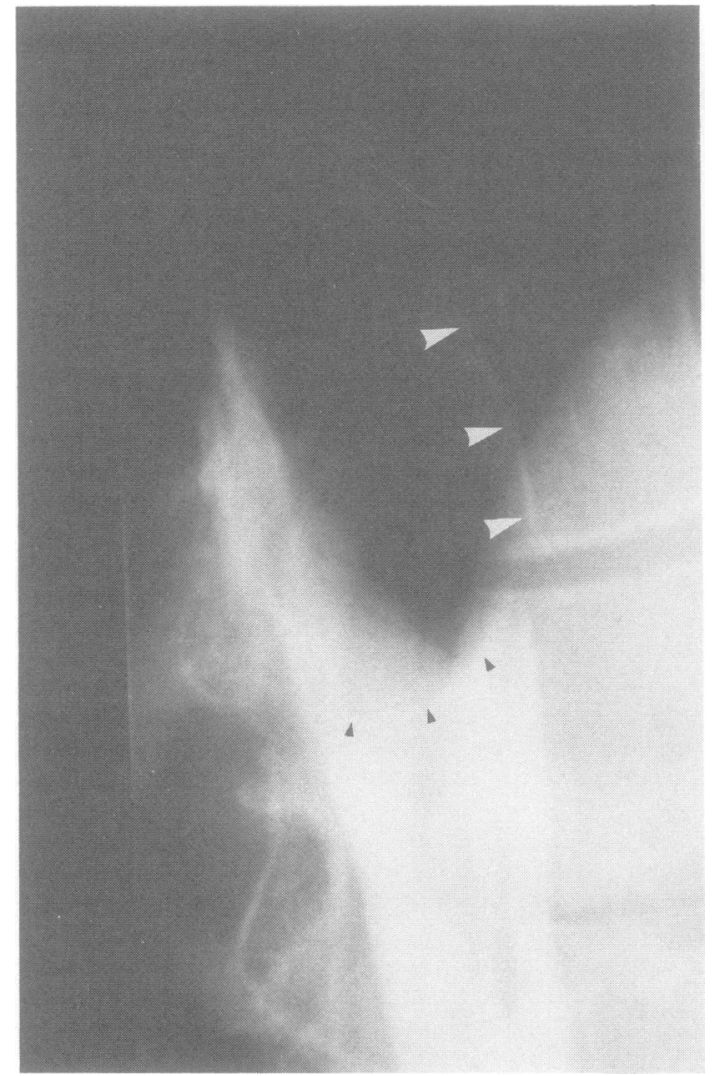

Fig 1 Lumbar metrizamide myelogram. "Complete" block with the sharp "cap" defect (black arrowheads) indicative of intradural extramedullary lesion. Scalloping of posterior surface of D12 vertebra (white arrowheads). 


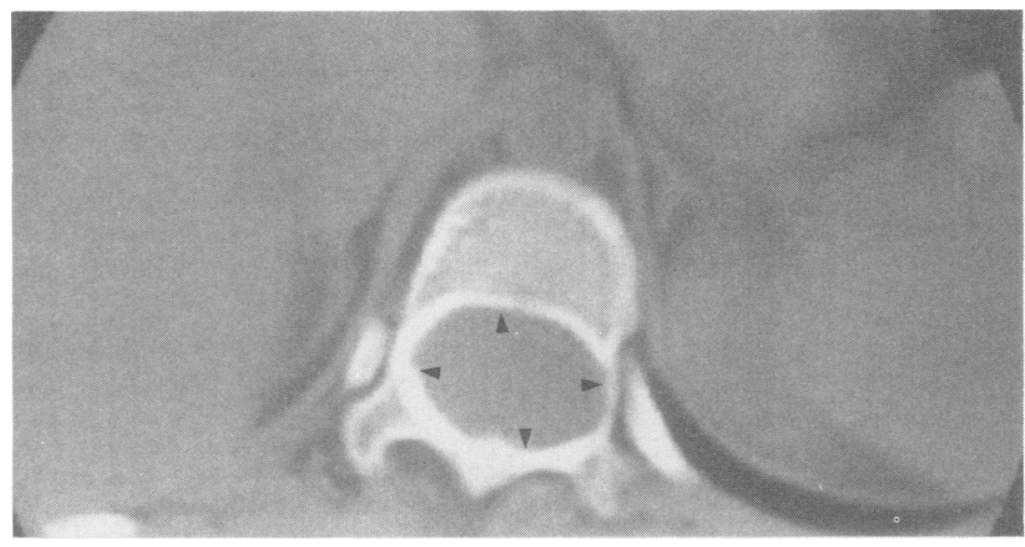

Fig 2 Postmyelography CT. Expansion of the spinal canal at D12 with thinning of the pedicle more on the left side.

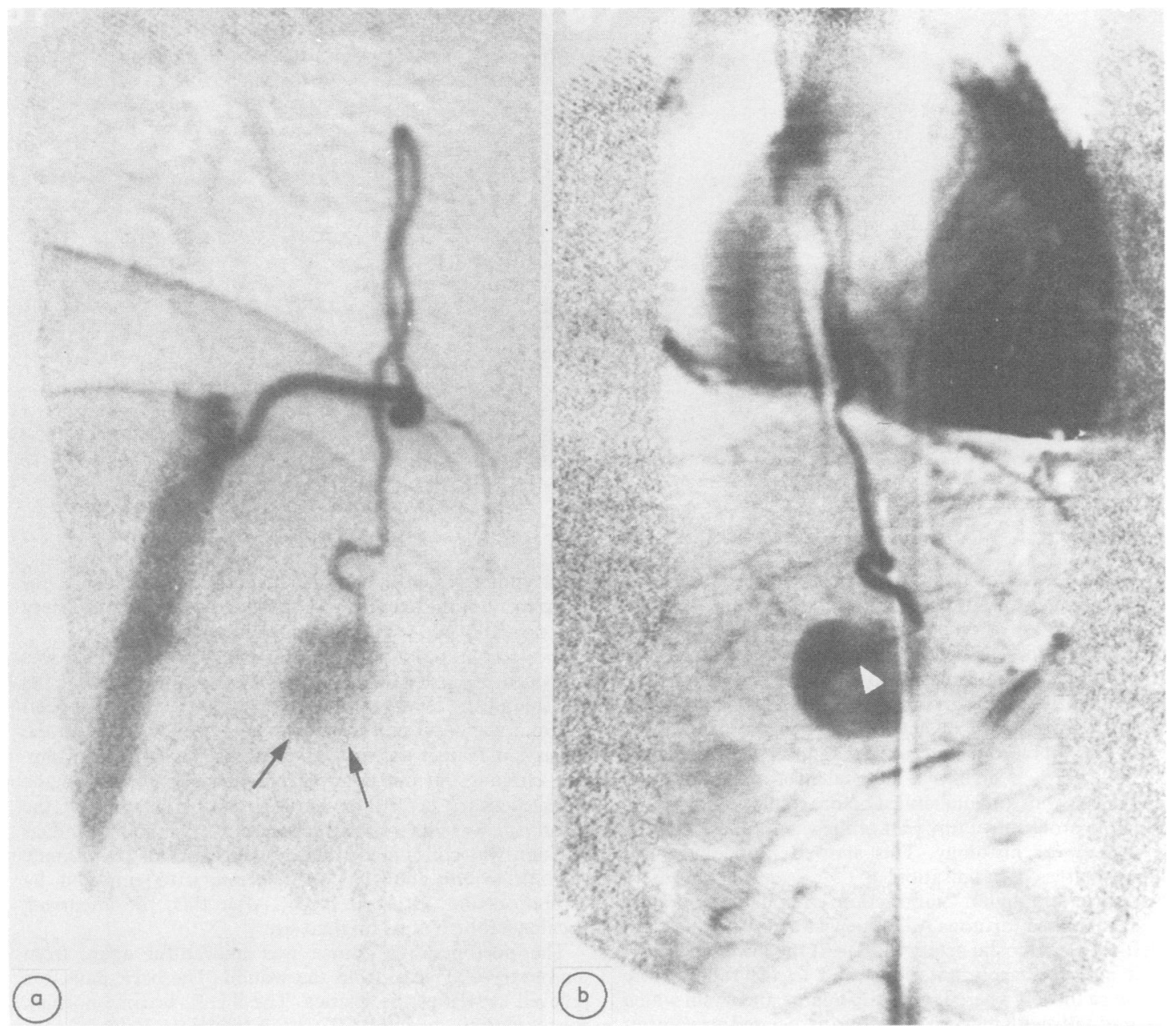

Fig 3 Spinal angiogram using digital subtraction. Gradual filling of large aneurysmal sac (arrows) by jet phenomenon (white arrowhead) from a dilated tortuous Adamkiewicz artery arising from left 10th intercostal artery. 

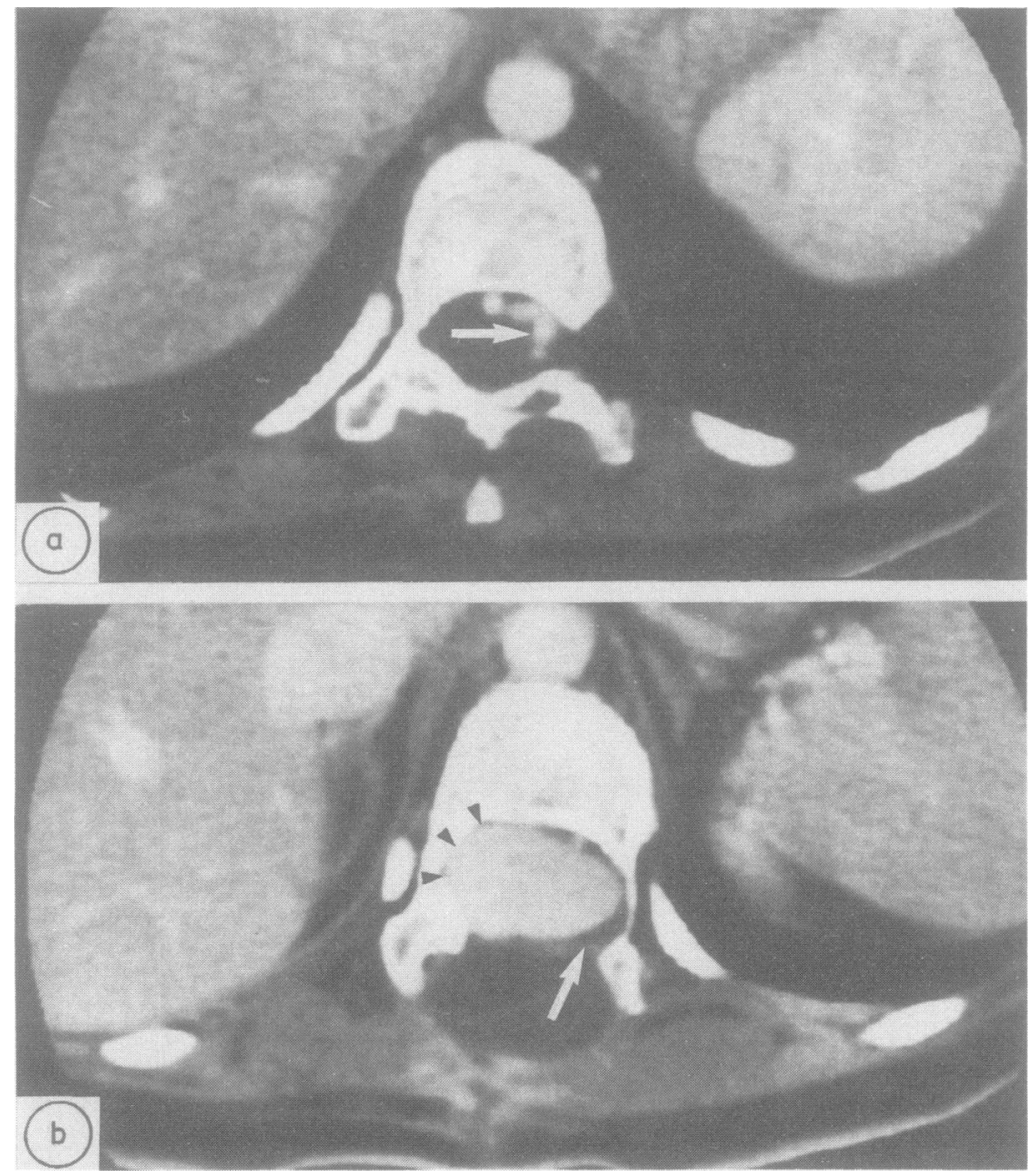

Fig 4 Dynamic CT with intravenous bolus of contrast medium. Dilated tortuous Adamkiewicz artery (arrow) at the level of lower border of L1 (a). Aneurysmal sac (arrowheads) occupying and expanding the spinal canal at D12, space occupied by the cord, as was seen at surgery (arrow) (b). Note postoperative changes and laminectomy of the first exploration.
The first operation was performed for excision of a possible S1 neurofibroma. Laminectomy from D12-L2 exposed the cause of block to be a swelling occupying the major part of the canal displacing the conus mainly posteriorly and to the left. The tumour felt cystic and was densely adherent to the conus over the whole of its left border. Attempts at dissection or aspiration were associated with profuse arterial bleeding. Once the diagnosis of an aneurysm was established, a definitive surgical procedure was considered hazardous and unjustified before spinal angiography. During the procedure a tiny part of the wall of the aneurysm was excised for histology. This showed fibrocollagenous tissue with areas of halinisation.

Intra-arterial digital subtraction spinal angiography revealed a dilated tortuous Adamkiewicz artery arising from the left 10th intercostal artery (fig 3). At the lower border of D11 it curved sharply anteriorly and to the right before crossing to the left where it ended in a large aneurysm which filled gradually with marked stasis. No associated arteriovenous malformation was seen. Noncontrast CT showed a hyperdense mass with wall calcification. Dynamic CT with intravenous bolus of contrast medium showed dense enhancement of the aneurysm. The dilated Adamkiewcz artery was seen above its lobulated upper margin (fig 4).

In a second operation, 3 weeks later, the same field was reopened. The artery of Adamkiewicz was approached on the left side of the cord, just before making its transverse bend where it was most accessible and as close as possible to the aneurysm. Here it was clipped, using a straight Yasargil clip. The clipping did not cause any ischaemic changes on the somatosensory evoked potential tracing. The size of the aneurysm was not obviously reduced by clipping, and as excision was considered hazardous because of its intimate relation to the conus, it was necessary to empty it by aspiration and letting it bleed. After this, the aneurysm became a fourth of its original size.

The postoperative course was uneventful apart from temporary CSF leak from the wound. The back pain was relieved as well as the sciatica. The S.L.R. became normal. Postoperative, dynamic CT with intravenous bolus of contrast, 1 month and again 1 year later, showed no filling of the aneurysm. A month later at follow up, the pain in the back 
and sciatica, as well as urgency of micturition were completely relieved.

\section{Discussion}

The rarity of spinal aneurysms is thought to be related to the small calibre of the spinal vessels and the infrequency with which they are affected by atherosclerosis.' In Djindjian's series of over 3000 spinal arteriograms, only one pure spinal aneurysm was encountered. ${ }^{6}$ The largest previously described spinal aneurysm measured $1.5 \times 0.8 \times 0.4 \mathrm{~cm}$, and presented as in our case, as an intradural mass lesion. ${ }^{4}$ In our case, however, the aneurysm was much larger, measuring $3 \times 3 \times 2.2 \mathrm{~cm}$, causing complete block on myelography. The dilatation of the Adamkiewicz artery suggested that this could be an aneurysmal varix of an arteriovenous fistula rather than a true arterial aneurysm, but no large draining veins were demonstrated in the spinal angiography series.

The symptoms dated back to early years of the patient's life. This long duration is also reflected on the associated bony changes (fig 1). The lesion was extramedullary but intimately related to the conus, compressing the conus and some roots on the right side giving radicular symptoms. Although the conus appeared radiologically to be markedly displaced and compressed, there was only minimal clinical evidence of pyramidal signs.
Dynamic CT with intravenous bolus of contrast medium should be helpful in the preoperative diagnosis of such cases. This is however not often considered due to the rarity of this presentation. Spinal angiography remains as the definitive examination.

\section{References}

1 Gracia CA, Dalcey S, Dalcey J. Ruptured aneurysm of the spinal artery of Adamkiewicz during pregnancy. Neurology 1979;29:394-8.

2 Henson RA, Croft PB. Spontaneous spinal subarachnoid haemorrhage. QJ Med 1956;25:53-66.

3 Kito K, Kobayashi N, Mori N, Kohno H. Ruptured aneurysm of the anterior spinal artery associated with pseudoxanthoma elasticum. Case report. J Neurosurg 1983;58:126-8.

4 Leech PJ, Stockes BAR, ApSimon T, Harper C. Unruptured aneurysm of the anterior spinal artery presenting as paraparesis. Case report. J Neurosurg 1976;45: 331-3.

5 Moore DW, Hunt WE, Zimmerman JE. Ruptured anterior spinal artery aneurysm. Repair via a posterior approach. Neurosurgery 1982;10:626-30.

6 Pia HW, Dijindjian R. Spinal angiomas: Advances in Diagnosis and Therapy. Berlin: Springer Verlag, 1987:117.

7 Stehbens WE. Pathology of the Cerebral Blood Vessels. St. Louis: CV Mosby, 1972:455-6.

8 Vincent FM. Anterior Spinal artery aneurysm presenting as hemorrhage. Stroke 1981;12:230-2. 